\title{
Influence of Different Weighting Materials on the Wear of N80 Metal Surface
}

\author{
Han Cheng $^{1}$, Qiu Zhengsong ${ }^{2}$, Yang Zhonghan ${ }^{1}$, Liu Xianyu ${ }^{1}, \mathrm{Yu} \mathrm{Yi}^{1}$ \\ ${ }^{1}$ CNOOC Ltd., Zhanjiang, Guangdong, China \\ ${ }^{2}$ College of Petroleum Engineering, University of Petroleum, Qingdao Shandong, China
}

Email address:

hancheng3@cnooc.com.cn (Han Cheng), hubeihancheng@163.com (Qiu Zhengsong), yangzhh9@cnooc.com.cn (Yang Zhonghan), liuxy19@cnooc.com.cn (Liu Xianyu),yuyi2@cnooc.com.cn (Yu Yi)

\section{To cite this article:}

Han Cheng, Qiu Zhengsong, Yang Zhonghan, Liu Xianyu, Yu Yi. Influence of Different Weighting Materials on the Wear of N80 Metal Surface. Advances in Materials. Vol. 5, No. 2, 2016, pp. 9-12. doi: 10.11648/j.am.20160502.11

Received: May 10, 2016; Accepted: May 31, 2016; Published: June 14, 2016

\begin{abstract}
Influence of high-density drilling fluid weighted with conventional barite, micronized barite, hematite and manganese tetroxide on the wear behavior of $\mathrm{N} 80$ metal surface was investigated. The worn surface morphologies and roughness of the N80 metal was observed and tested respectively by scanning electron microscope and surface roughness meter tester. The wear mechanisms of different weighting materials were also discussed. It was found that the erosion rate of N80 steel worn by micronized barite is $57.8 \%$ of it worn by conventional barite, and the erosion rate of N80 steel worn by manganese tetroxide is $43.8 \%$ of it worn by hematite. The main wear mechanism of conventional barite, micronized barite, hematite and manganese tetroxide on the metal surface is abrasive wear, plowing, planning action and surface fatigue wear, under conditions of high density drilling fluid. Manganese tetroxide and micronized barite can significantly reduce the wear on the metal surface because of filling and adsorption on the concave and convex of the metal surface, especially the roughness of N80 steel surface worn by manganese tetroxide can be reduced to $50 \%$ of the hematite.
\end{abstract}

Keywords: Weighting Materials, Micronized Barite, Manganese Tetroxide, Wear, High-Density Drilling Fluid

\section{Introduction}

Casing wear is related to drilling fluid, dogleg severity, contact force between drill pipe and casing, and casing steel grade. Weighting material is one of the main factors influencing the casing wear. At the same time, with the development of deep and ultra-deep drilling technology, high-density drilling fluid are widely applied increasingly, the abrasion problems of traditional high-density drilling fluid weighting materials (API barite and hematite) on drill string and casing are becoming more and more prominent [1-4]. Scholars at home and abroad have carried out some researches on function of drilling fluid during the abrasion process. Recently Liang used different steel grade casings to be tested in drilling fluids with different densities. It was found that with the increasing of drilling fluid density, the casing wear rate decreased quickly and then increasing gradually [5]. On the basis of the casing wear prediction model, the effects of dogleg angle, casing steel grade and drilling fluid performance on the casing wear could be quantitatively analyzed by Yang. The results show that the casing wear depth increases with the increase of dogleg angle, but decreases with the increase of drilling fluid density [6]. But the studies on casing wear are mainly for API barite and hematite.

Micronized barite and manganese tetroxide has become more and more popular weighting material for high density drilling fluid. However, the study of micronized barite and manganese tetroxide on drilling tools and casings are less [7-8]. This paper reviews the influence of high-density drilling fluid with conventional barite, micronized barite, hematite and manganese tetroxide on the wear behavior of N80 metal surface. The wear mechanism of four weighting materials on metal surface will be discussed.

\section{Experiment Part}

\subsection{Instruments and Materials}

Instruments: ML-10 abrasive wear testing machine, RM-20 
pocket surface roughness meter, electronic balance, Hitachi S-4800 cold field emission scanning electron microscope.

Materials: 1) Drilling fluid additive: Drilling fluid bentontie was provided by Weifang Huawei Bentonite Group Co., Ltd, China, following the API standard. $\mathrm{NaOH}$ was purchased from Sinopharm Chemical Reagent Co., Ltd, China. PAC-lv,
SMP-1, SMC, FT-A and Lube was provided by Huaxin Mud Additives Co., Ltd, China.

2) Weighting materials: micronized barite, conventional barite, manganese tetroxide, hematite. The basic parameters of four weighting materials are listed in Table 1.

Table 1. Basic parameters of weighting materials.

\begin{tabular}{|c|c|c|c|c|c|c|c|}
\hline \multirow{2}{*}{ weighting materials } & \multirow{2}{*}{ Chemical component } & \multirow{2}{*}{$\operatorname{density} /\left(\mathrm{g} / \mathrm{cm}^{3}\right)$} & \multirow{2}{*}{ Moh's hardness } & \multicolumn{3}{|c|}{ Particle size distribution/ $\mu \mathrm{m}$} & \multirow{2}{*}{$\begin{array}{l}\text { Special surface } \\
\text { area } /\left(\mathrm{m}^{2} / \mathrm{g}\right)\end{array}$} \\
\hline & & & & $D_{10}$ & $\mathbf{D}_{50}$ & $\mathrm{D}_{90}$ & \\
\hline conventional barite & $\mathrm{BaSO}_{4}$ & 4.21 & 3.2 & 1.585 & 18.24 & 52.62 & 0.488 \\
\hline micronized barite & $\mathrm{BaSO}_{4}$ & 4.30 & 3.1 & 0.777 & 2.491 & 8.302 & 2.963 \\
\hline hematite & $\mathrm{Fe}_{2} \mathrm{O}_{3}$ & 5.15 & 6.3 & 6.851 & 43.12 & 146.7 & 0.187 \\
\hline manganese tetroxide & $\mathrm{Mn}_{3} \mathrm{O}_{4}$ & 4.82 & 5.6 & 0.765 & 1.149 & 2.721 & 4.761 \\
\hline
\end{tabular}

The scanning electron microscope observations of four weighting materials are shown in Figure 1.
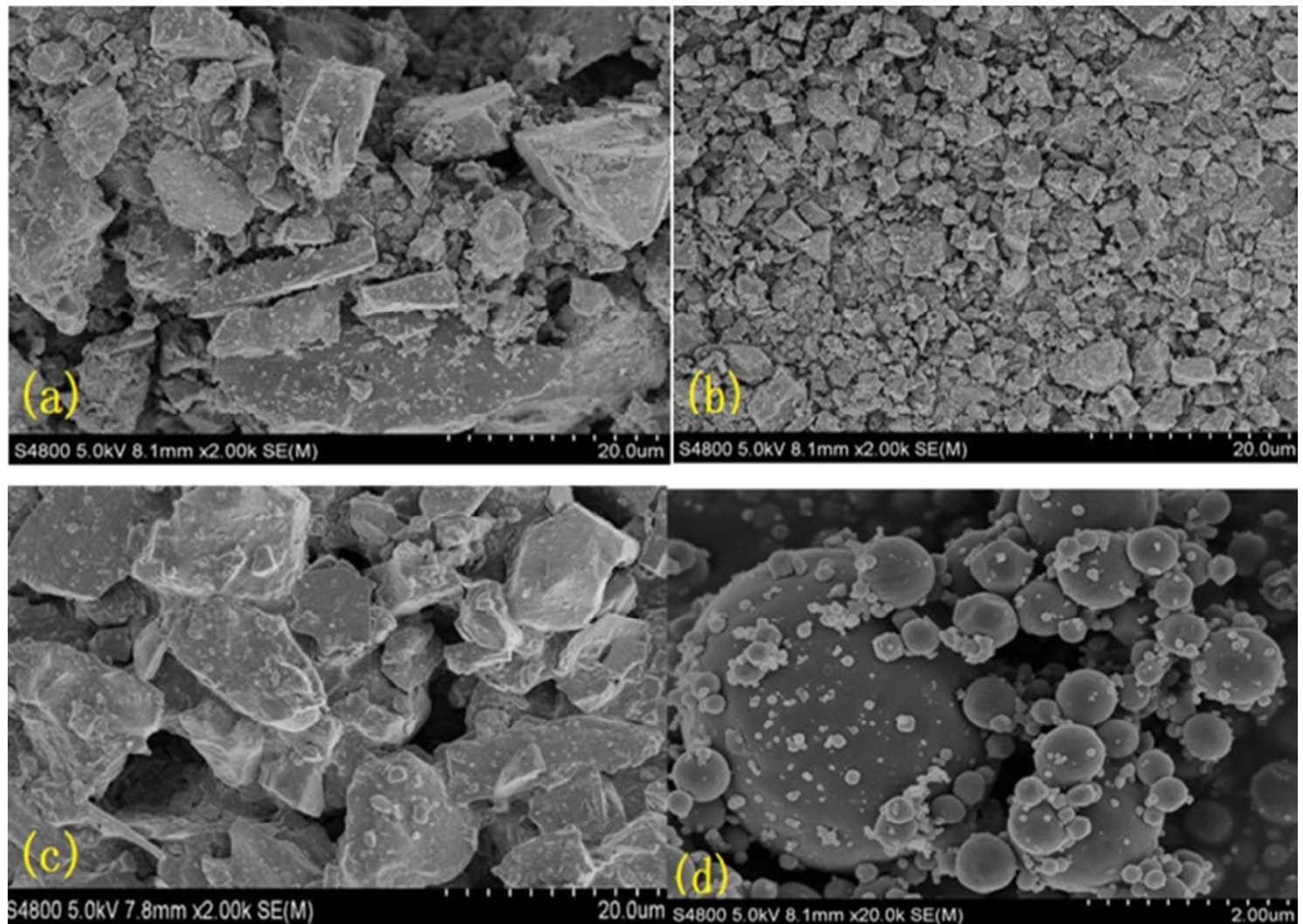

Figure 1. SEM photographs of four weighting materials: (a) conventional barite (2000 times), (b) micronized barite (2000 times), (c) hematite (2000 times), (d) manganese tetroxide (5000 times).

\subsection{Experiment Design}

1) The formula of polymer sulfonated drilling fluid from ultra-deep drilling: $4 \%$ bentonite $+0.3 \% \mathrm{NaOH}+0.3 \% \mathrm{PAC}-\mathrm{lv}$ $+4 \%$ SMP-1 $+3.5 \%$ SMC $+2.5 \%$ FT-A $+2 \%$ Lube, drilling fluids' densities are increased to $2.0 \mathrm{~g} / \mathrm{cm}^{3}$ by using micronized barite, conventional barite, manganese tetroxide and hematite respectively. After hot rolling at $150^{\circ} \mathrm{C}$ for $16 \mathrm{~h}$, these testing fluids were stirred fully and used for next the next experiment.

2) ML-10 abrasive wear testing machine is used to test the influence of the above testing fluids on casing surface abrasion. Followed by spindle, the disc-shaped samples rotate and friction emerges by scratching indenter. Its working principle is shown in Figure 2.

Disc-shaped sample and scratching indenter were made of N80 casing material and S135 drill pipe material respectively. Based on the drilling conditions, the rotation speed of turntable was set at $60 \mathrm{r} / \mathrm{min}$, the contact pressure between disc-shaped sample and scratching indenter is $25 \mathrm{~N}$, and the speed of indenter moving along turntable is $4 \mathrm{~mm} / \mathrm{s}$. During the experiment, four groups of high-density drilling test fluid weighted by conventional barite, micronized barite, hematite and manganese tetroxide are poured into turntable. After running $8 \mathrm{hrs}$, four N80 steel disc-shaped samples are washed and dried. 


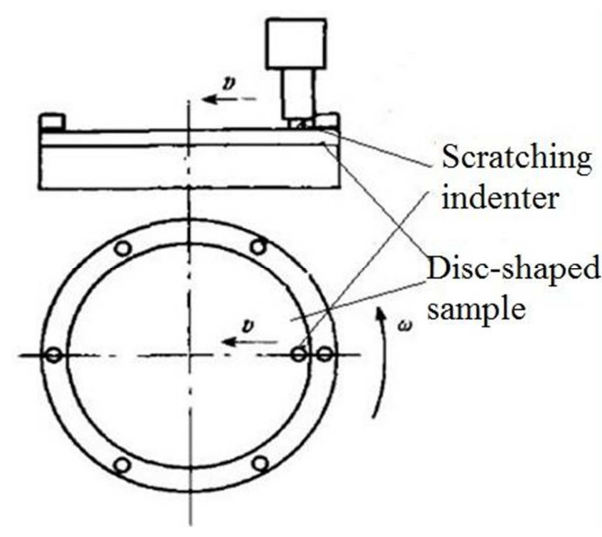

Figure 2. Working principle of $M L-10$ abrasive wear testing machine.

\section{Results and Discussion}

\subsection{The Influence of Weighting Materials on Wear Value}

The wear values of four N80 steel disc-shaped samples are shown in Figure 3. As Figure 3 shows, the order of abrasion degree of high-density drilling fluid by adding different weighting materials to N80 steel from high to low is conventional barite, hematite, micronized barite and manganese tetroxide. The wear values of N80 steel by micronized barite is $57.8 \%$ of conventional barite; yet the wear values of $\mathrm{N} 80$ steel by manganese tetroxide is $43.8 \%$ of hematite. It is obvious the abrasion degree of manganese tetroxide and micronized barite to N80 steel are significantly lower than traditional high-density weighting materials.

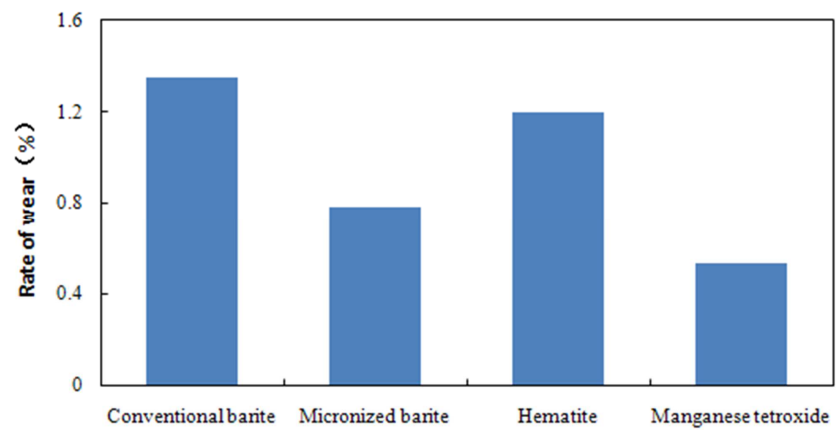

Figure 3. Influence of different weighting materials on N80 steel abrasion consumptions.

\subsection{Influence of Weighting Materials on Metal Surface Abrasion}

After worn in test fluids weighted by different weighting materials, the surface scanning electron microscope results of N80 steel disc-shaped sample are shown in Figure 4. Figure 4(a) shows that there are large number of long and continuous abrasive indentation, wide furrows and pits and grooves in different sizes on the surface of N80 steel sample, after worn in conventional barite-based drilling fluid. This is due to the grooves and cracks being plowed on the surface of conventional barite during friction. Metal materials are flaking away, mainly characterized by abrasive wear [9-10].

Figure 4(b) shows that the grooves and pits on the surface of
N80 steel samples are less than conventional barite, after worn in micronized barite-based drilling fluid. Figure 1(a) and Figure 1(b) shows that conventional barite particles are long shapes, while the majority of micronized barite particles are blocky shapes. The size of micronized barite particle is significantly smaller than conventional barite particle, and thus it has higher particle sphericity, the plowing effect on the metal surface is significantly smaller than conventional barite in the process of friction.

Figure 4(c) shows that there are large number of long, deep and continuous pits and grooves on the surface of N80 steel sample, after worn in hematite-containing drilling fluid. And bottom of the grooves is smooth, and appears plastic fracture and flake. This is due to the high hardness of hematite particles (Moh's hardness is 6.3), which have large particle size, irregular polyhedron and sharp edges (as show in Figure 1(c)). Thus the grooves are planed on the surface of wear sample by hematite particles directly during friction [11-12].

Figure 4(d) shows that, compared with the high-density drilling fluid containing conventional weighting materials barite and hematite, wear degree of N80 steel samples in high-density drilling fluid by adding manganese tetroxide is less. The surface of the samples has plastic deformation and horseshoe-shaped shallow pit, followed with shallow scratches, indentations. In Figure 1 (d), manganese tetroxide is spherical particles, and the surface of sample is squeezed repeatedly by manganese tetroxide particles during friction. Fatigue cracks form and particles detach, which result in shallow pits and indentation. Thus it gives rise to surface fatigue wear.

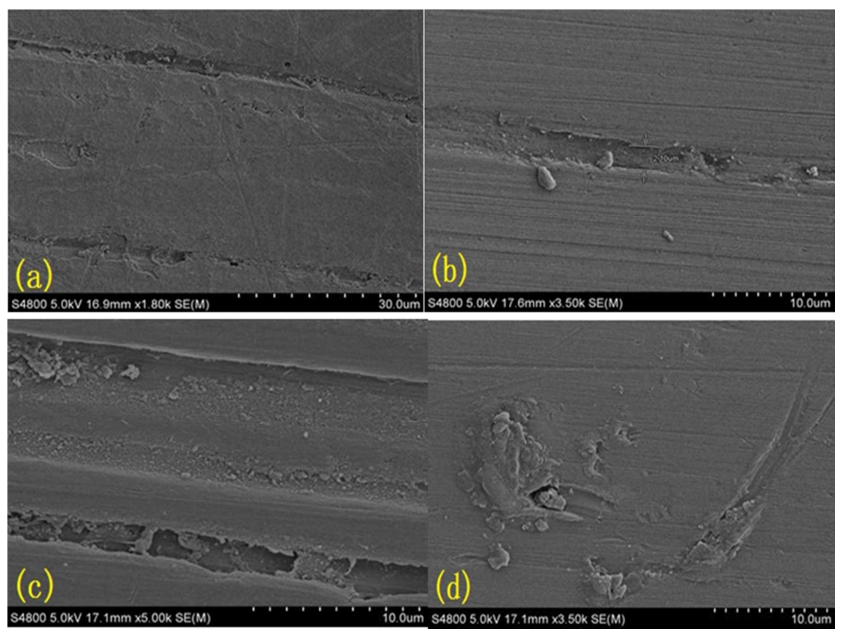

Figure 4. SEM photographs of sample surface after wear in different experiment pulp by adding different weighting materials: (a) conventional barite (1800 times), (b) micronized barite (3500 times), (c) hematite (5000 times), (d) manganese tetroxide (3500 times).

\subsection{Influence of Weighing Materials on the Roughness of Metal Surface}

RM-20 pocket surface roughness meter is used to test the surface roughness of disc-shaped samples in experiment pulp by adding different weighting materials before and after the wear, the results are shown in Figure 5. After wear in test 
fluids weighted by different weighting materials, the roughness of sample surface increase at certain levels. Ra and $\mathrm{Rz}$ of N80 steel disc-shaped samples are around $50 \%$ of hematite-containing test fluid after wear in manganese tetroxide-containing test fluid, the influence of micronized barite on the roughness of sample surface is less than conventional barite.

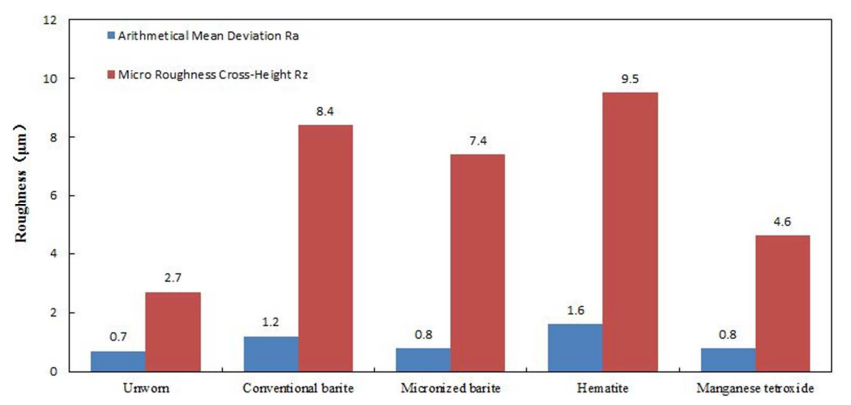

Figure 5. Surface roughness of N80 steel in experiment pulp by adding different weighting materials.

Results analysis: Figure 5 shows that before abrasion, Ra on the surface of N80 steel disc-shaped sample is $0.7 \mu \mathrm{m}, \mathrm{Rz}$ is 2.7 $\mu \mathrm{m}$, and the average particle size of manganese tetroxide and micronized barite is $1.149 \mu \mathrm{m}$ and $2.491 \mu \mathrm{m}$, respectively. The particle sizes are no different with the roughness of sample surface, which make the particles tend to fill in the concave and convex of N80 steel disc-shaped sample surface; while the special surface area of manganese tetroxide and micronized barite particles is $4.761 \mathrm{~m}^{2} / \mathrm{g}$ and $2.963 \mathrm{~m}^{2} / \mathrm{g}$ respectively. The large special surface area makes the particles easily adsorbed on the concave and convex surface of N80 steel disc-shaped sample [13]. The fill and absorption function of manganese tetroxide and micronized barite on metal concave and convex surface are shown in Figure 6 The fill and adsorption function on metal surfaces and higher degree of particles sphericity could reduce the cutting action on the metal surface during friction, and thus the roughness of sample in the experiment pulp by adding manganese tetroxide and micronized barite is lower after wearing. The particle size of conventional barite and hematite are larger and the shapes are irregular, so the roughness of samples in conventional barite and hematite is larger after wearing.

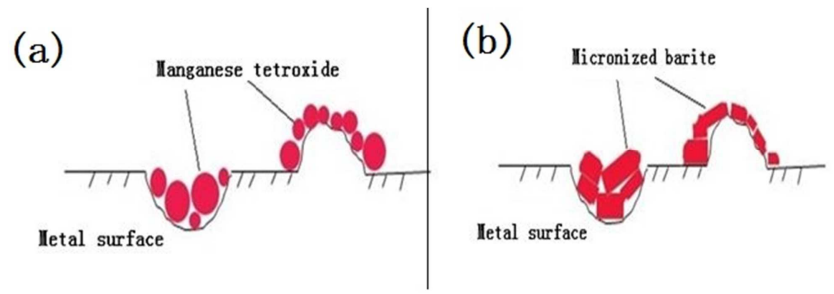

Figure 6. Reaction of manganese tetroxide and micronized barite on the metal concave and convex surface: (a) Manganese tetroxide, (b) Micronized barite.

\section{Conclusion}

The abrasion of conventional barite and micronized barite are mainly particle abrasion. Compared with conventional barite, the particle size of micronized barite is smaller and the sphericity is higher, which could reduce abrasion. The wear mechanism of metal surface by hematite is planning and manganese tetroxide is fatigue. The spherical particles could significantly reduce the abrasion of metal surface.

The abrasion of manganese tetroxide and micronized barite can be reduced on metal surface due to their small particle size, large special surface area and easy fill and absorption on N80 steel concave and convex surface.

\section{References}

[1] Dong X. J., Yang Z. F., He W. T. Study and Development of Casing Wear. China Petroleum Machinery, 2008, 37 (4): 32-36.

[2] Hou Y. J., Wang, W. W. Study Evolution of Casing Wear. Drilling \& Production Technology, 2001, 24 (5): 72-74.

[3] Tehrani A., Cliffe A., Hodder M. H. Alternative Drilling Fluid Weighting Agents: A Comprehensive Study on Ilmenite and Hematite., SPE 167937, IADC/SPE Drilling Conference and Exhibition, Fort Worth, Texas, USA, March, 2014.

[4] Dou Y. H., Jiang X. H., Zhang F. X. Experimental Research on Casing Wear in Drilling Fluids of High Density. Lubrication Engineering, 2007, 32 (9): 26-28.

[5] Liang E. G., Li Z. F, Wang J. M., et al. Experimental Study on Casing Wear Mechanism in Oil and Gas Wells. Petroleum Drilling Techniques, 2015, 43 (1): 69-73.

[6] Yang C. X., Sun M. G., Tang H. L. Casing Wear Prediction and Casing Protection Technology for Extended Reach Well. China Petroleum Machinery, 2016, 44 (1): 5-9.

[7] Han X. M, Fan J. C., Zhang L. B. Experimental Study on the Effects of Drilling Fluid Viscosity on Casing Wear. Drilling Fluid \& Completion Fluid, 2007, 24 (4): 63-65.

[8] Yan L. L., Wang J. H., Xu X. G. Study on Erosive and Magnetic Effects of Weighting Agent in a High Density Water-Based Drilling Fluid, OTC 24787, Offshore Technology Conference-Asia, Kuala Lumpur, Malaysia, March, 2014.

[9] Quercia G., Belisario R., Rengifo R. Reduction of erosion rate by particle size distribution (PSD) modification of hematite as weighting agent for oil based drilling fluids. Wear, 2009, 266 : $1229-1236$

[10] Yu L., Zhang L. B., Fan J. C. Influence of Hematite and Barite on the Friction and Wear Behavior of Casing and Drill Pipe Pair. Tribology, 2004, 24 (5): 462-466.

[11] Gonzáleza J. M., Quinteroa F., Arellanoa J. E. Effects of interactions between solids and surfactants on the tribological properties of water-based drilling fluids. Colloids and Surfaces A: Physicochemical and Engineering Aspects, 2011, 391: 216-223.

[12] Xiao G. Z., Zhao G. X., Han Y. Experiments on the abrasion of casings by barite and hematites. Drilling Fluid \& Completion Fluid, 2007, 24 (5): 74-75.

[13] Wang P., Lin, K., Wang L. Study on wear properties of heavy agent of drilling mud. Oil Field Equipment, 2005, 34 (2): 27-30. 